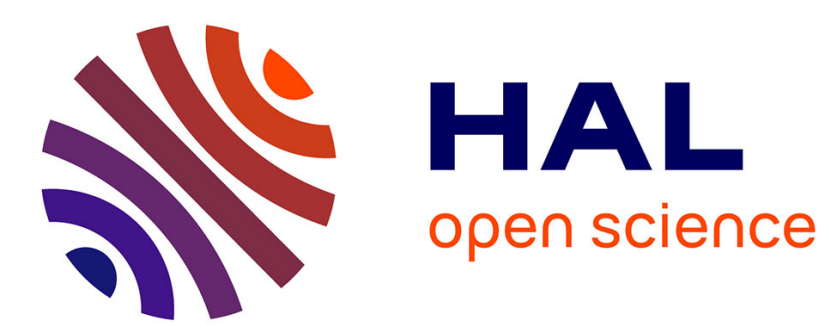

\title{
Observation de défauts cristallins en microscopie électronique à balayage
}

\author{
M. Pitaval, P Morin, J. Baudry, G. Fontaine
}

\section{To cite this version:}

M. Pitaval, P Morin, J. Baudry, G. Fontaine. Observation de défauts cristallins en microscopie électronique à balayage. Journal de Physique Lettres, 1976, 37 (11), pp.309-312. 10.1051/jphyslet:019760037011030900 . jpa-00231299

\section{HAL Id: jpa-00231299 https://hal.science/jpa-00231299}

Submitted on 1 Jan 1976

HAL is a multi-disciplinary open access archive for the deposit and dissemination of scientific research documents, whether they are published or not. The documents may come from teaching and research institutions in France or abroad, or from public or private research centers.
L'archive ouverte pluridisciplinaire HAL, est destinée au dépôt et à la diffusion de documents scientifiques de niveau recherche, publiés ou non, émanant des établissements d'enseignement et de recherche français ou étrangers, des laboratoires publics ou privés. 
Classification

Physics Abstracts

$7.116-7.166$

\title{
OBSERVATION DE DÉFAUTS CRISTALLINS EN MICROSCOPIE ÉLECTRONIQUE A BALAYAGE
}

\author{
M. PITAVAL, P. MORIN, J. BAUDRY et G. FONTAINE \\ Département de Physique des Matériaux (*), \\ Université Claude Bernard Lyon I, 43 Bd du 11 Novembre 1918, 69621 Villeurbanne, France
}

(Reçu le 26 juillet 1976, accepté le 15 septembre 1976)

\begin{abstract}
Résumé. - Dislocations et fautes d'empilement sont observées en microscopie électronique à balayage soit en transmission, soit par réflexion sur des films minces. Sur des échantillons massifs les dislocations sont visualisées en filtrant les électrons rétrodiffusés.
\end{abstract}

Abstract. - Dislocations and stacking faults are observed in Scanning Electron Microscopy in transmission or reflection from thin films. For thick samples, dislocations are observed when slow backscattered electrons are removed by an energy filter.

1. Introduction. - On sait que pour observer des défauts cristallins avec un faisceau d'électrons, on opère généralement en transmission sur des lames préalablement amincies. Le but de cette lettre est de présenter des observations préliminaires effectuées en réflexion et de montrer la possibilité d'opérer sur des échantillons massifs. Pour ce but nous avons utilisé un microscope électronique à balayage (M.E.B.) sur lequel nous avons notamment adapté un canon à émission de champ et un filtre d'énergie qui agit sur les électrons rétrodiffusés. Ces observations peuvent se comparer à la méthode de Berg et Barrett couramment utilisée par réflexion de rayons $\mathrm{X}$. La résolution est ici bien plus grande puisqu'elle est de l'ordre de la distance d'extinction (quelque $100 \AA$ ) à comparer aux quelque $10 \mu \mathrm{m}$ des rayons $X$. Par contre la profondeur explorée sous la surface du cristal est plus faible, de l'ordre de quelques distances d'extinction.

2. Principe. - En M.E.B., quand on balaye la surface polie d'un échantillon monocristallin à l'aide d'un faisceau parallèle d'électrons $\left(\theta_{\mathrm{i}} \leqslant 10^{-3} \mathrm{rad}\right.$.), on observe des anomalies sur l'intensité des électrons rétrodiffusés (par exemple), chaque fois que le faisceau incident se présente sous l'angle de Bragg pour une série donnée de plans cristallographiques [1]. On obtient ainsi des pseudo-lignes de Kikuchi dont l'interprétation géométrique est évidente et dont l'intensité se calcule en théorie dynamique à $N$ ondes [2]. De telles lignes sont observées figure 4

(*) Associé au C.N.R.S.

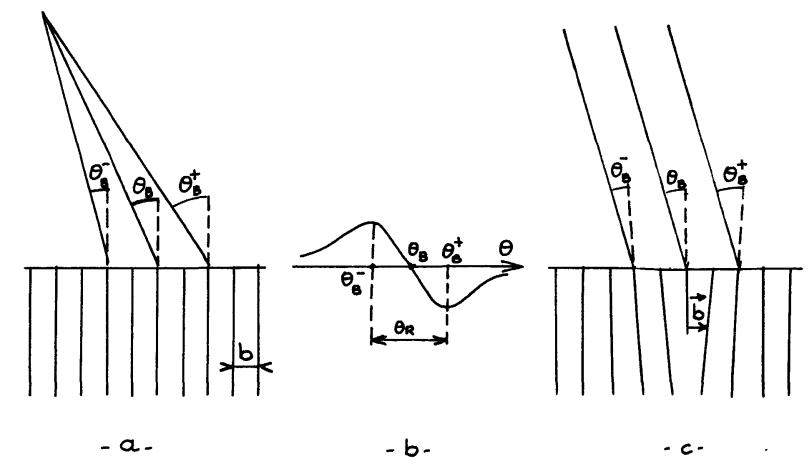

FIG. 1. - Origine du contraste sur une dislocation (voir texte) $a$ ) balayage par un faisceau parallèle d'électrons; $b$ ) intensité rétrodiffusée ; c) observation à fort grandissement d'une dislocation.

sur du silicium. A deux ondes la figure $1 b$ donne les caractéristiques de l'intensité observée et calculée pour les positions de sonde de la figure $1 a$. Comme $\xi_{\mathrm{g}} \simeq 300 \AA$ pour des faibles indices, la largeur angulaire des lignes correspondantes

$$
\theta_{\mathrm{R}}=\frac{b}{\xi_{\mathrm{g}}} \simeq 10^{-2} \mathrm{rad}
$$

La figure $1 c$ montre qu'à fort grandissement (on peut négliger la variation d'incidence de la sonde) on peut s'attendre à un contraste analogue pour une dislocation. La largeur $\delta$ de l'image est telle que la rotation des plans cristallographiques produite par la dislocation à cette distance soit environ $b / 2 \pi \delta$ égale $\theta_{\mathrm{R}}$. Cette discussion sommaire, qu'on peut rendre plus quantitative [3], montre que pour observer 
un contraste de dislocations par réflexion, il faut balayer l'échantillon avec une sonde électronique d'ouverture $\theta_{\mathrm{i}}<\theta_{\mathrm{R}}$ et de taille $d<\delta$ donc de brillance

$$
B=\frac{4 I}{\pi^{2} d^{2} \theta_{\mathrm{i}}^{2}}>\frac{16 I}{b^{2}} \simeq 10^{16} I .
$$

En pratique, compte tenu des faibles contrastes observés, un rapport signal sur bruit convenable impose un courant de sonde minimum $I \simeq 10^{-9} \mathrm{~A}$. On est donc amené à construire une sonde de brillance $B \gtrsim 10^{7} \mathrm{~A} \mathrm{~cm}^{-2} \mathrm{sr}^{-1}$, c'est-à-dire un canon à électrons utilisant l'émission de champ (un canon ordinaire a une brillance au plus égale à $\left.10^{5} \mathrm{~A} \mathrm{~cm}^{-2} \mathrm{sr}^{-1}\right)$.

3. Réalisation. - Nous avons construit un canon à émission de champ que nous avons adapté sur l'objectif d'un microscope à balayage conventionnel. Les caractéristiques de ce dispositif ayant été décrites ailleurs [4], nous nous limiterons à en résumer les principales.

L'émetteur est une pointe de tungstène d'orientation (111) formée sous champ à $1700 \mathrm{~K}$ pour augmenter l'intensité du faisceau, puis chauffée à $1200 \mathrm{~K}$ pendant l'émission pour rendre négligeable sur plus de 4 heures la dérive de l'intensité [5]. Pour éliminer les fluctuations résiduelles de l'émission, le signal vidéo est asservi à l'intensité du faisceau détectée sur le diaphragme d'objectif.

La chambre objet a été modifiée pour pouvoir observer des défauts cristallins sur des échantillons minces ou massifs. Pour les échantillons minces un détecteur placé sous l'échantillon capte les électrons transmis à travers un diaphragme centrable. Des bobines de balayage placées entre l'échantillon et le diaphragme permettent d'obtenir des clichés de diffraction sur des zones de diamètre inférieur à $1000 \AA$. Par déplacement du diaphragme, on peut observer l'objet en champ clair ou sombre sur tache sélec-

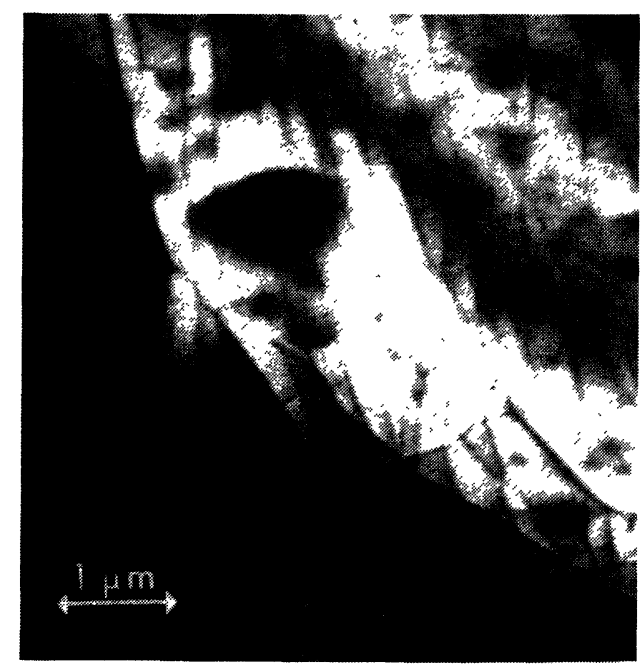

(1) tionnée. Simultanément, on peut former l'image avec les électrons rétrodiffusés. Pour les échantillons épais, un filtre d'énergie a été construit pour éliminer les électrons rétrodiffusés dont la profondeur d'échappement est trop grande et qui diluent tout signal dû aux couches superficielles [8]. Canon et filtre fonctionnent jusqu'à $45 \mathrm{kV}$. La résolution en énergie du filtre est de l'ordre de $1 \%$. Les résultats qui suivent ont été pris dans ces conditions.

4. Résultats. - La figure $2 a$ représente un réseau de dislocations observé en transmission sur une lame mince de $\mathrm{MoS}_{2}$. La figure $2 b$ représente la même région observée avec les électrons rétrodiffusés. Les contrastes et les contours d'extinction sont complémentaires en première approximation. En fait, une étude détaillée des contrastes est difficile car la rotation de notre porte-objet n'est pas très précise. La figure $3 a$ représente une faute d'empilement observée en transmission sur une lame mince d'acier inoxydable. La figure $3 b$ représente la même région observée avec les électrons rétrodiffusés. Les contrastes sont encore complémentaires. Comme pour les dislocations, on ne voit que la partie supérieure de la lame jusqu'à trois ou quatre distances d'extinction. La figure 4 illustre l'intérêt du filtre d'énergie utilisé pour observer les échantillons massifs à l'aide des électrons rétrodiffusés. Il s'agit de pseudo-lignes de Kikuchi sur $\mathrm{du}$ silicium. La figure $4 a$ correspond à des électrons rétroduiffusés non filtrés et la figure $4 b$ à des électrons filtrés. De plus le signal de la figure $4 a$ a été traité pour renforcer le contraste (niveau de noir) et la figure $4 b$ non. On voit que l'utilisation du filtre renforce le contraste notamment des lignes fines de hauts indices qui sont au centre de la zone A. Plus important : le filtrage supprime une bonne partie de la dissymétrie des lignes (voir par exemple les lignes B) qui est donc liée à l'absorption [2]. Finalement la figure 5 est le premier exemple de dislocations

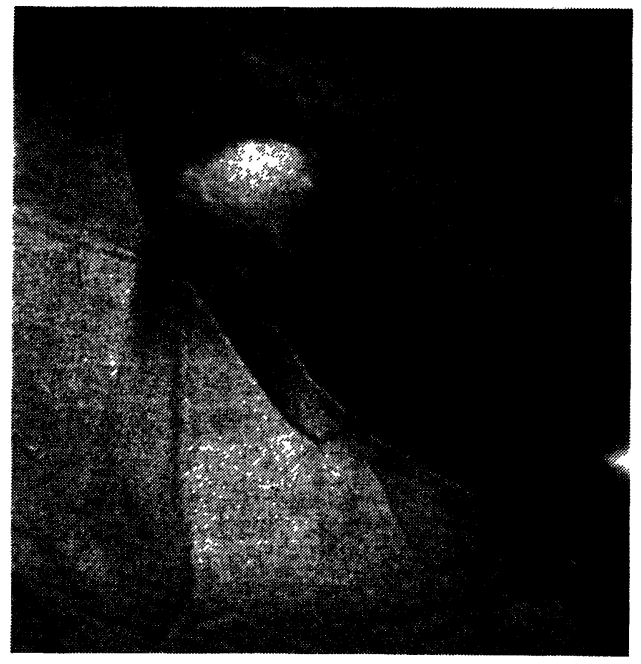

b)

FIG. 2. - Dislocations $a$ ) en transmission, $b$ ) en réflexion $\left(\mathrm{MoS}_{2}\right)$. 


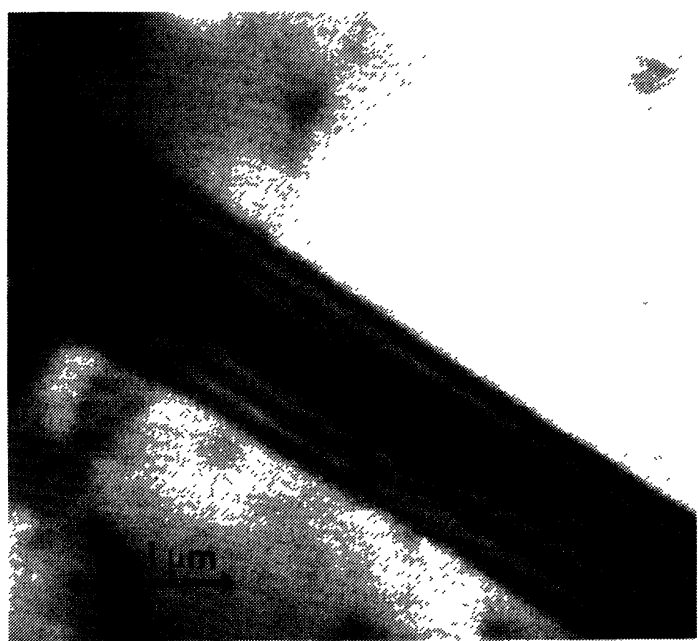

(a)

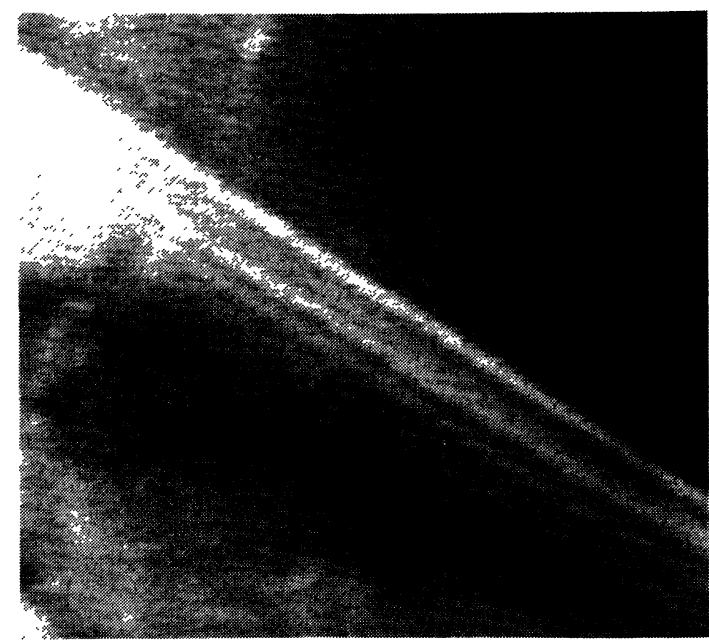

b)

FIG. 3. - Fautes d'empilement (acier inoxydable) : $a$ ) en transmission, $b$ ) en réflexion.

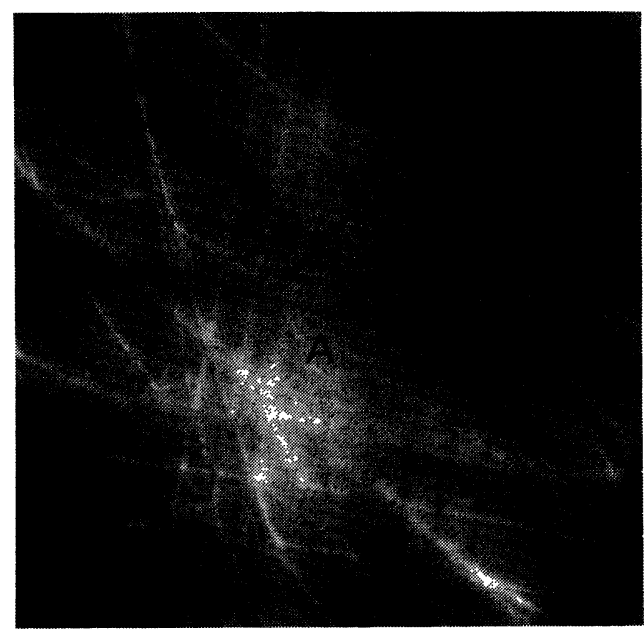

a)

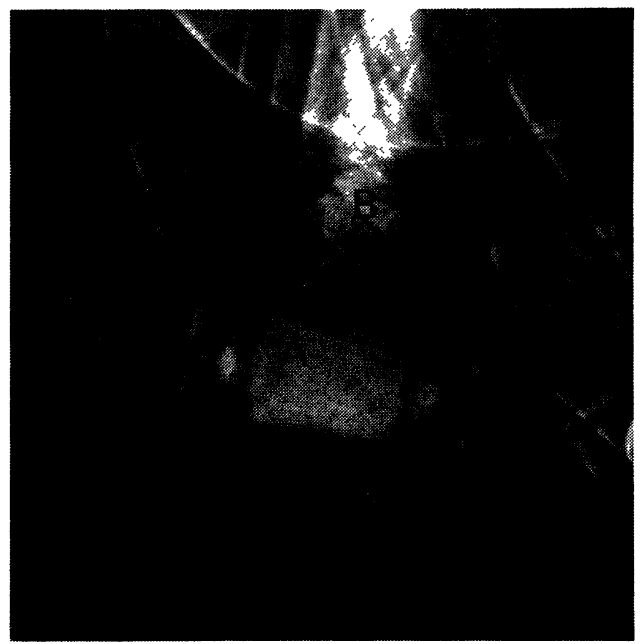

b)

Fig. 4. - Pseudo-lignes de Kikuchi électrons rétrodiffusés $a$ ) non filtrés, $b$ ) filtrés.

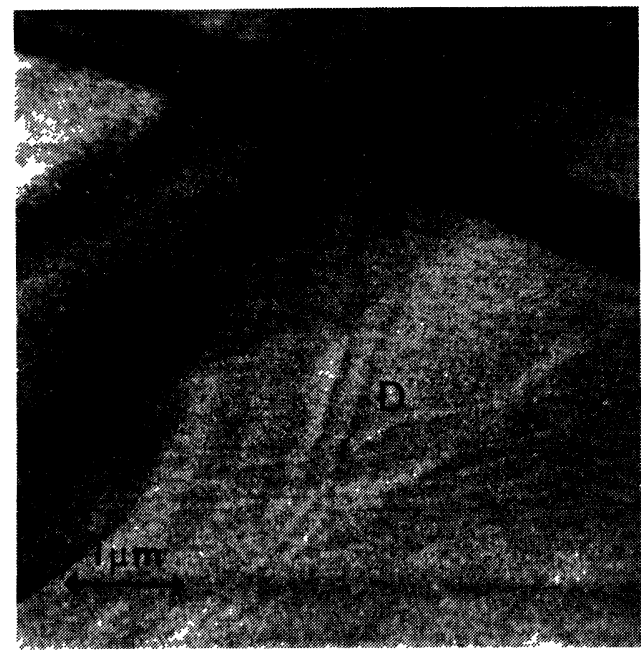

FIG. 5. - Dislocations sur un échantillon massif, $\left(\mathrm{MoS}_{2}\right)$ image filtrée.

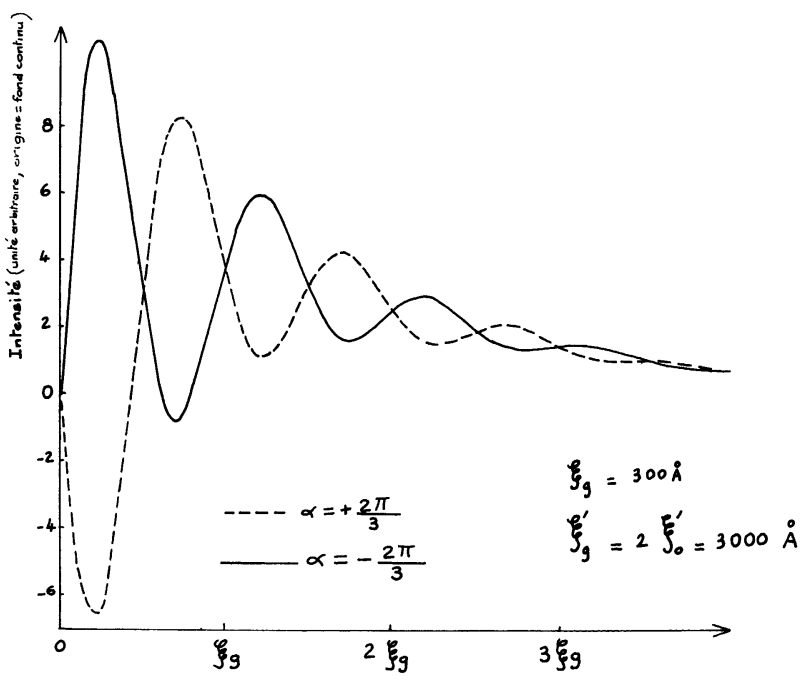

Fig. 6. - Calcul de contrastes sur une faute d'empilement inclinée à $45^{\circ}$ par rapport à la surface. 
observées sur un échantillon massif à l'aide des électrons rétrodiffusés filtrés. Le réseau $D$ de dislocations se trouve au voisinage d'une marche de clivage $\mathrm{M}$. On peut le comparer à ce qu'on observe sur une lame mince (Fig. 2b). Résolution et contraste ne sont pas encore aussi bons mais nous pensons pouvoir les améliorer en augmentant la résolution du filtre.

5. Conclusion. - Les constrastes des images de défauts cristallins obtenus avec les électrons rétro- diffusés sur des cristaux épais se calculent assez simplement en théorie dynamique [3]. Il suffit de généraliser la théorie donnant le contraste des pseudolignes de Kikuchi [2]. A titre d'exemple la figure 6 donne le contraste calculé pour une faute d'empilement dans un C.F.C. suivant le déphasage $\alpha=2 \pi \mathbf{g} . \mathbf{R}$ introduit par le défaut. On constate que la frange de bord est brillante ou sombre suivant que $\alpha$ est négatif ou positif. Ce contraste peut se comparer à l'observation de la figure $3 b$. Il est bien complémentaire du contraste observé en transmission $[6,7]$.

\section{Bibliographie}

[1] Coates. D. G.. Phil. Mag. 16 (1967) 1179.

[2] Vicario, E., Pitaval, M., Fontaine, G., C. R. Hebd. Séan. Acad. Sci. B270(1970) 1402.

[3] Clarke, D. R., Howie, A., Phil. Mag. 24 (1971) 959.

[4] Pitaval, M., Morin, P., Baudry, J., Fontaine, G.. Developments in Electron Microscopy and Analysis (Proc. EMAG 75 Venables, J. A., Editor Academic Press) 1975, 19.
[5] Ranc, S., Pitaval, M., Fontaine, G., Surf. Sci. 1976 (à paraître).

[6] Stern, R. M., Ichinokawa, T., Takashima, S., Hashimoto, H., Kıмото, S., Phil. Mag. 26 (1972) 1495.

[7] Thompson, M. N., S.E.M. (The Institute of Physics Edt.) 1973, 176.

[8] Wells, O. C., Appl. Phys. Lett. 19 (1971) 232. 